\title{
Shielding of the NBI Boxes against W7-X Magnetic Stray Fields
}

Manfred Kick (1), Juliusz Sielanko (2), Bernd Heinemann (1), Rudolf Riedl (1), Eckehart Speth (1), Albrecht Stäbler (1)

1. Max-Planck-Institut für Plasmaphysik, Boltzmannstr. 2, D-85748 Garching, Germany

2. Maria Curie Sklodowska University, PI. M. C. Sklodowskie 1, 20-031 Lublin, Poland

\section{Abstract:}

Neutral Beam Injection (NBI), besides ECRH, is foreseen as one of the main heating devices at the W7-X stellarator currently under construction at IPP Greifswald, Germany. In a final stage $20 \mathrm{MW}$ of NBI heating power will be installed generated by two NBI boxes of the AUG type. Since magnetic fields generally affect the trajectories of charged particles, essentially all the NBI boxes -including ion sources, acceleration sections, neutralisers and deflection magnetsmust be shielded against the stray fields of W7-X

In the magnetic stray fields of W7-X there exist significant radial and toroidal components whereas at tokamaks the vertical components are dominant. The power loads on the ion dump and the protecting structures of the deflecting magnets and the beam lines caused by residual beam ions, therefore, will be strongly different. Thus the shielding concept of AUG cannot simply be taken over, but must be carefully redesigned in order to remain below the critical power limits.

New modelling calculations of the magnetic shielding, the ion trajectories and the resulting power loads have been carried out for the "high iota" and "low shear" experimental scenarios of W7-X. The fields taken for these calculations are modelled by averaging the calculated W7-X stray fields on the one hand, and by fields generated by two -hypothetical- planar coils perpendicular to the $x-y$ plane, on the other hand.

The shielding concept for W7-X mainly consist of iron plates in the outer side regions of the boxes and as little magnetic material as possible inside the boxes. 
Keywords:

Neutral Beam Injection, W7-X stellarator, W7-X stray fields, shielding of magnetic fields, PROFI code, ion dump, residual ions

Introduction:

NBI produces neutral particles of high energies for plasma heating in magnetic confinement fusion devices, 50 to $93 \mathrm{keV}$ at ASDEX Upgrade (AUG), $55 \mathrm{keV}$ at W7-X (100 keV in a later stage). Besides the desired particles, which need to be neutral in order to penetrate the plasma confining magnetic fields, charged particles are produced by NBI, too. Residual ions, i.e. not neutralised or re-ionised beam ions, carry a large fraction (in the order of one half) of the power generated in the ion sources. In the case of W7-X NBI, as at AUG, undesired powers of about 2.5 MW per source (i.e. $25 \mathrm{MJ}$ in a $10 \mathrm{~s}$ beam pulse) are generated and need to be dissipated in a controlled way on cooled structures. The residual ions, therefore, are directed to ion dump panels by deflecting magnets. "Stray fields" of stellarators or tokamaks, i.e. their "external" magnetic fields, in the regions between ion sources and deflecting magnets cause deflections of the ion trajectories compared to the undisturbed ones. These ions may lead to damages in the NBI boxes and/or the beam lines if large power loads are deposited in areas not adequately cooled. The stray fields, therefore, need to be carefully shielded, in order to prevent uncontrolled depositions of power.

\section{Objectives:}

At W7-X start-up NBI with $10 \mathrm{MW}$ of heating power (4 RF ion sources, 2 in each of the 2 boxes) will be installed at module 2 of W7-X [1], [2]. $20 \mathrm{MW}$ of NBI are foreseen in a later stage (4 additional ion sources). The axes of the beam boxes are rotated at $r=6.75 \mathrm{~m}$ (W7-X polar coordinates) in poloidal direction compared to the radial direction by $+/-7.4441^{\circ}(\rightarrow$ balancing of co/counter-injected particles), fig.1. The NBI system will mainly be a copy of the AUG NBI [3], [4] with ion sources of the PINI type [5], [6].

The magnetic fields of the W7-X stellarator in the NBI box regions have been calculated for various experimental scenarios, e.g. standard (i.e. pure stellarator field generated by the non planar coils, no additional poloidal components), high iota (hi iota), or low shear [7]. In fig. 2 
a contour plot of the W7-X field in the $x-y$ plane $(z=+305 \mathrm{~mm})$ for the hi iota scenario is shown (different colours indicate different values of the magnetic field, the arrows indicate the field directions, their lengths are not to scale!). In comparison to the axisymmetric tokamak field of AUG, the W7-X stellarator field decreases faster with radius. Whereas at AUG mainly the large component of $B$ in the $z$ direction needs to be shielded (by iron side plates in the deflecting magnet region, iron yokes on the magnet, central iron rips in the neutraliser region and the ion source flanges with gate valve housings and neutraliser shieldings, magnetically connected to the iron housing for the ion sources), at W7-X a concept for reducing the influence of all three components, r-, $\varphi$ - and z-, must be found for the different experimental scenarios with their markedly different stray fields. For the standard and hi iota scenarios all the components of $B$ in the NBI box region are nearly equal, whereas for hi iota the $B_{r}$ - and $B_{z^{-}}$components are slightly larger. For low shear the $B_{r}$ component is about one half, the $B_{\varphi}$ component is about one fourth compared to hi iota, whereas $B_{z}$ is slightly larger.

From the layout of the ion dump components and the experience at AUG NBI maximum power loads of $2500 \mathrm{~W} / \mathrm{cm}^{2}$ on the ion dump panels (brazed Cu-Cr-Zr, water cooled), and $300 \mathrm{~W} / \mathrm{cm}^{2}$ on the inertially cooled other target structures are regarded as upper limits. The residual magnetic fields inside the shielded volumes must guarantee that the deposited powers on the various target structures do not exceed these values. Since the calculations show that even very small residual fields inside the shieldings do not always guarantee an appropriate power deposition, not the size of the residual magnetic fields, but the deposited powers have been taken as criteria for the rating of the shielding.

From calculations and measurements in the ion source areas of ASDEX-Upgrade a criterion of $0.3 \mathrm{mT}$ had been derived for the size of the magnetic field inside the ion source shielding house [8]. Since the stray fields of W7-X decrease much more rapidly with distance than at AUG (BW7$x / B_{A U G} \approx 1 / 4-1 / 5$ at the extraction grids) the shielding house design has been regarded to be adequate and thus not been changed. Calculations of the field distribution in the shielding house area of W7-X confirm this assumption resulting in values below $0.2 \mathrm{mT}$, which, from the experience of AUG, is low enough not to affect the properties of the ion sources concerning 
uniformity, alignment, emittance, etc. significantly. At the extraction grids the stray field, $B_{W 7-X}$, without shielding is small, anyway, below $2 \mathrm{mT}$ for all components.

\section{Results}

The magnetic field distributions inside the NBI boxes are calculated with the PROFI code [9], where the magnetic material of flanges, adapter tubes, gate valve housings, etc., is taken into account. A 3-D Monte Carlo code, based on the TRHN program [10], is used for calculating the ion trajectories and the resulting power density profiles on the various targets. Space charge effects, which widen the power deposition, are not taken into account. Accompanying calculations show that they reduce the maximum loads, as expected. Fig. 3a) shows the geometry, as taken for the PROFI calculations. The positions of the 4 possible ion sources are shown in fig. 3 b). For the calculations the racetrack shaped ion sources and neutralisers are approximated by rectangular ones. Calculations have been carried out for the ion sources Q1 and Q2, fig. 3b), of both beam boxes, NI $20\left(\varphi \approx 57^{\circ}, z=-305 \mathrm{~mm}\right)$, and NI $21\left(\varphi \approx 87^{\circ}, z=+305 \mathrm{~mm}\right)$.

A strong constraint to the shielding concept is the demand of minimising the total amount of iron, because this may affect the carefully optimised W7-X field in the plasma confinement region. Plates of $5 \mathrm{~cm}$ of iron on the side walls of the boxes, magnetically connected to the iron made shielding house of the ion sources and to iron plates in front of the deflecting magnet (looking from W7-X to the magnet), see fig. 4a), turn out to shield the influence of the W7-X field onto the ion trajectories very effectively, which holds if the thickness of the shielding plates is reduced to $3 \mathrm{~cm}$. In PROFI geometry the whole space is segmented into "cells" (not equidistant in real geometry) for mathematical reasons. A sketch of the possible conversion of this shielding to real geometry is shown in fig. $4 \mathrm{~b})$.

The W7-X field in the complete region of the beam boxes has been calculated by E. Harmeyer [7], [11] and M. Köppen [7], [12] with a cell size of $25 \mathrm{~cm}$ (in W7-X geometry) for the hi iota, low shear and standard cases.

Since the positions of the NBI boxes (axes) are symmetric to $\varphi=72^{\circ}$, and the W7-X field is equally symmetric, the stray fields in the regions of the two NBI boxes are symmetric, as well: $B_{r}$, though, changes its sign, whereas $B_{\varphi}$ and $B_{z}$ remain unchanged, see fig. 2. Calculations for 
$\mathrm{NI} 21$, therefore, can be carried out with NI 20 geometry, but with the appropriately changed $\mathrm{B}_{\mathrm{r}}$ (reversed sign).

For the PROFI code calculations the W7-X field is modelled in two different ways, firstly by averaging $B_{x}, B_{y}, B_{z}$ in the regions between the ion sources and the deflecting magnet, and secondly by two planar coils perpendicular to the $r-\varphi$ plane and the box axes. Both approximations lead to comparable results. The positions of the planar coils are indicated in fig. 5a), for the hi iota case, by blue lines (Coils $C 1$ and $C 2$ ). The fit to the real field, $B_{x}, B_{y}, B_{z}$, in the plane $z=-305 \mathrm{~mm}(\mathrm{NI} 20)$ is shown in fig. $5 b)$.

Since the beam axes are inclined with respect to the box axes, see fig. 3 b), the ion trajectories are influenced in a fairly large volume between the ion sources and the magnets (x-direction), and between the ion source and the box axis (z-direction), with beam dimensions added on. In order to ensure, that the approximation reasonably fits the real fields of both NBI boxes the modelled fields have been examined in various cuts of this volume, showing reasonable agreement in the entire range.

With these modelled fields the resulting magnetic fields in the region of the boxes, the ion trajectories and the power loads on the different panels are calculated. Fig. 6a) shows the field distribution for NI 21 (hi iota case) in the region of the beam box with shielding, with the current of the deflecting magnet turned on. The colour code is the same as in the field plots of figs. 2 and 5. The iron masses of the ion source flanges, ion source gate valve housings and adapter tubes (for the valves and the neutralisers) are included in the calculations. The necessary openings in the shielding plates for the steering mechanisms of the ion source gate valves are also taken into account. The field inside the boxes (outside the magnet) is small (essentially below $50 \mathrm{G}$ ). Inside the shielding material it remains in the range of 1000 - $5000 \mathrm{G}$, i.e. below saturation, fig. 6a). The resulting power load on the main ion dump panel is shown as a contour plot in figs. 6 b). Its maximum remains with $1970 \mathrm{~W} / \mathrm{cm}^{2}$ well below the critical value of 2500 $\mathrm{W} / \mathrm{cm}^{2}$. The maximum loads on the -inertially cooled- outer and inner magnet pole shoe shielding panels remain below 10 and $50 \mathrm{~W} / \mathrm{cm}^{2}$, respectively. The same holds for $\mathrm{NI} 20$ and low shear scenario $\left(\mathrm{W}_{\max }<1660 \mathrm{~W} / \mathrm{cm}^{2}\right)$. Also for $\mathrm{NI} 20$, hi iota case, the power load on to the main ion dump remains below $1550 \mathrm{~W} / \mathrm{cm}^{2}$ (fig. 7). Even the low shear case for NI 20 with the slightly higher value of $2110 \mathrm{~W} / \mathrm{cm}^{2}$ (see fig. 8) does not exceed the maximum power load of 2500 
$\mathrm{W} / \mathrm{cm}^{2}$. Eventually, the $\mathrm{W} 7-\mathrm{X}$ standard case, i.e. reduced $\mathrm{Bz}$, has been examined with the result that in this the case, too, the maximum power loads remain well below the critical values. A good magnetic coupling, e.g. from iron plates inside the boxes to outside ones turned out to be crucial. The calculations show that this good coupling in the ion source flange region, i.e. at lower stray fields, is more important than in the deflection magnet region with its higher fields. Therefore, the shielding plates on the sides of the boxes have been placed outside the boxes, where a direct connection to the ion source shielding house is possible. The magnetic connection in the deflecting magnet region across the stainless steel box wall to the outside is made by large iron plates, facing each other, fig. 4 . In further examinations the side shielding plates have been reduced in thickness from $5 \mathrm{~cm}$ to $3 \mathrm{~cm}$, thus reducing their mass of iron by $2 / 5$. The power loads, thereby, were only somewhat increased. I.e. plates of $3 \mathrm{~cm}$ will be sufficient to get the desired shielding.

Further calculations have shown that iron made neutraliser tubes, as used on AUG, influence the fields in a way that the trajectories are more strongly focussed onto the main ion dump, thus increasing its power load. A magnetic connection of the ion source flange to the shielding also increases the power loads on the ion dumps.

\section{Conclusion}

A shielding concept for the W7-X NBI has been developed using vertical iron shielding plates ( 3 or $5 \mathrm{~cm}$ thick) on the outer sides of the boxes with a good magnetic coupling to the ion source shielding house, but "magnetically insulated" from the ion source flanges. Iron plates inside the boxes in front of the deflecting magnet (looking from W7-X to the magnet) are magnetically coupled across the stainless steel box walls to the outside placed side plates by large iron made facing areas. Side plates with a thickness of $3 \mathrm{~cm}$ turned out to produce the desired shielding. The PROFI and trajectory calculations with modelled W7-X stray fields show in the examined cases of the W7-X high iota, standard and low shear scenarios maximum power loads below the critical values of $2500 \mathrm{~W} / \mathrm{cm}^{2}$ for the water cooled structures, and $300 \mathrm{~W} / \mathrm{cm}^{2}$ for the inertially cooled ones. The magnetic fields inside the shielded volume which provide these results are reduced to values below $0.2 \mathrm{mT}$ in the ion source and acceleration grid regions, below $1 \mathrm{mT}$ in the largest part of the 
neutraliser even behind the ion source flange and only in a small neutraliser region closest to the magnet they get into the order of $2 \mathrm{mT}$, see Figs. $6,7,8$.

Small magnetic field values in the shielded volumes, however, do not provide automatically appropriately distributed deposited powers. Even lower fields in certain parts of the box may result in higher and/or inappropriately deposited powers on the target structures. Hence, not the reduced field values, but the reduced and appropriately distributed deposited powers have been taken as the main criterion for the layout of the magnetic shielding.

\section{Acknowledgements}

The authors like to express their thanks to E. Harmeyer and M. Köppen for accomplishing the calculations of the W7-X field in the region of the NBI boxes and to N. Rust and P. Mc Neely for many fruitful discussions.

\section{References:}

1. V. Erckmann, H.-J. Hartfuß, M. Kick, et al., IEEE (1998), p. $40-48$

2. H. Renner, J. Boscary, V. Erckmann, et al., Nuclear Fusion, 40, No.6, (2000) p. $1083-1093$

3. A. Stäbler, O. Vollmer, J.H. Feist et al., 18th SOFT (1994), vol. 1 (1995), p. 593 - 596

4. O. Vollmer, A. Stäbler, E. Speth et al., 20th SOFT (1998) p. 449

5. G. Düsing, H. Altmann, H. Falter et al., Fusion Technology, 11 (1987), p. 163

6. E. Speth, M. Ciric, JH. Feist et al., Fusion Engineering and Design, 46 (1999) p. 383-388

7. T. Andreeva, E. Harmeyer, IPP Report 13/2 (2003)

8. P. Franzen , S. Obermayer, J. Schäffler, A. Stäbler, E. Speth, O. Vollmer, Fusion Engineering and Design 56-57 (2001) p. 487-491

9. W. Müller, Electrical Engineering (Archiv für Elektrotechnik), 69, 5, (1986) p. 295-300

10. A. Stäbler, J. Sielanko, S. Götz, E. Speth, Fusion Technology 26, 2 (1994), p. 145

11. Harmeyer, E. private communication

12. Köppen, M., private communication 


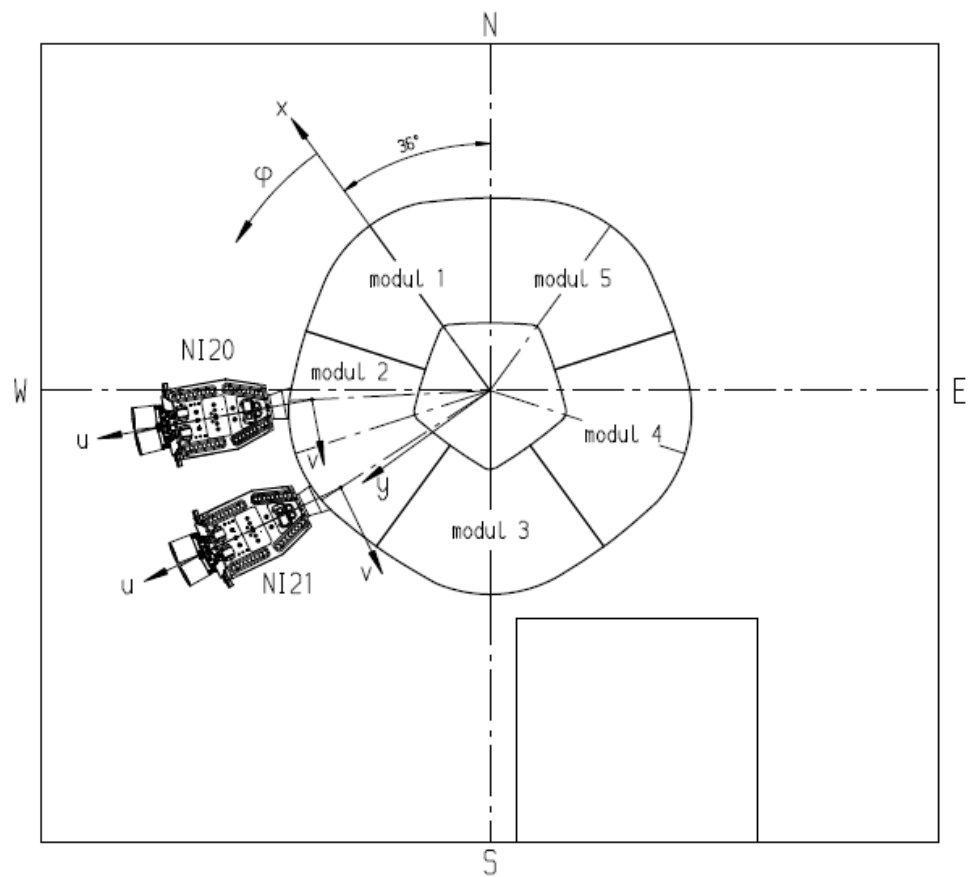

Definition of coordinate systemes

- cartesian coordinate system of $W 7-X(x, y, z)$

- polar coordinate system of $W 7-X(\varphi, r, z)$

- coordinate systems of NBI-boxes $(u, v, w)$

NI20

\begin{tabular}{|c|c|c|}
\hline$u=0$ & $\varphi=56.9059^{\circ}$ & $x=3685.61$ \\
\hline$v=0$ & $r=6750$ & $y=5654.98$ \\
\hline$w=0$ & $z=-305$ & $z=-305$ \\
\hline
\end{tabular}

NI21

\begin{tabular}{|c|c|l|}
\hline$u=0$ & $\varphi=87.0941^{\circ}$ & $x=342.19$ \\
\hline$v=0$ & $\Gamma=6750$ & $y=6741.32$ \\
\hline$w=0$ & $z=+305$ & $z=+305$ \\
\hline
\end{tabular}

Fig.1: Top view of W7-X with NBI boxes NI20 and NI 21, coordinates:

W7-X: $x, y, z$ and $\varphi, r, z$,

NBI Boxes: u, v, w

87.1

$|\mathrm{B}|[\mathrm{mT}]$ in $\mathrm{Z}=+0.305 \mathrm{~m}$ (High lota)

$\mathrm{B}[\mathrm{mT}]$

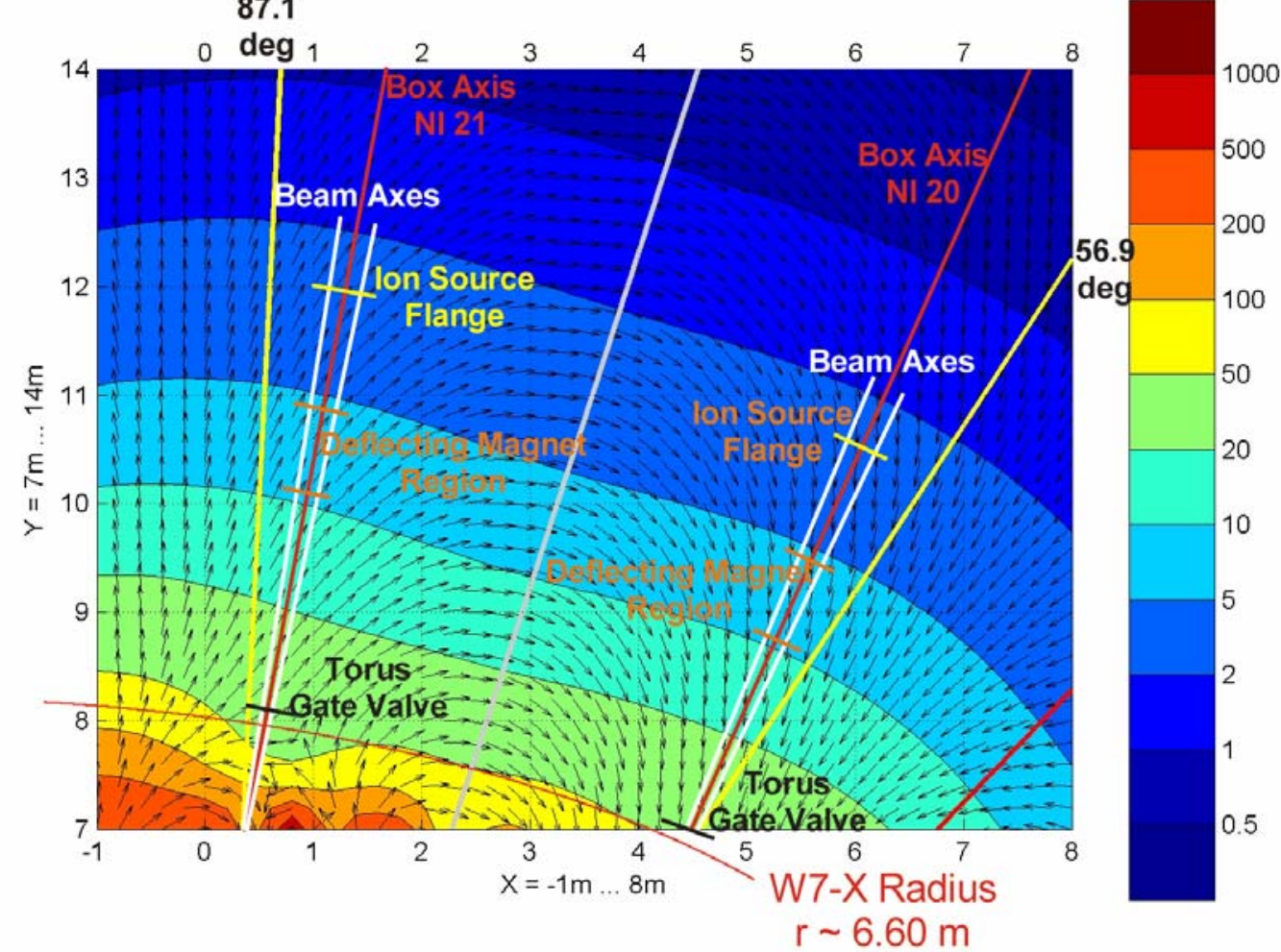

Fig. 2: W7-X stray field in the region of NBI, the approximate positions of the box axes (red), beam axes (white) and ion source flanges (yellow), deflecting magnet regions (orange) and torus valves (black) are indicated; colour code for $B(m T)$, see right; the arrows show the directions of $\mathrm{B}$ (lengths not to scale) 


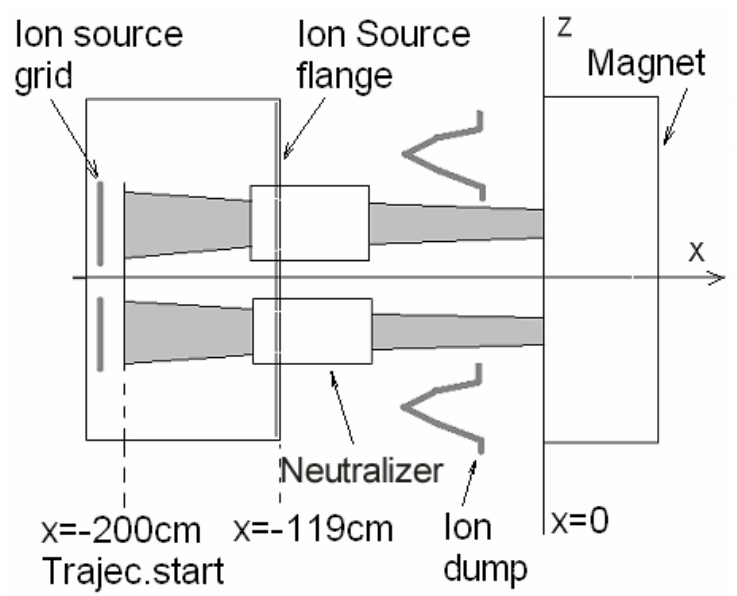

Fig. 3: Geometry of the PROFI calculations

a) Positions of the grids, ion source flange and neutraliser, deflecting magnet and beam dumps; the position of the ion sources is left of the grids $x=$ PROFI coordinate (are not to scale, but dependent on the cell size of the calculations),

$x=0$ indicates the "entrance of the magnet"

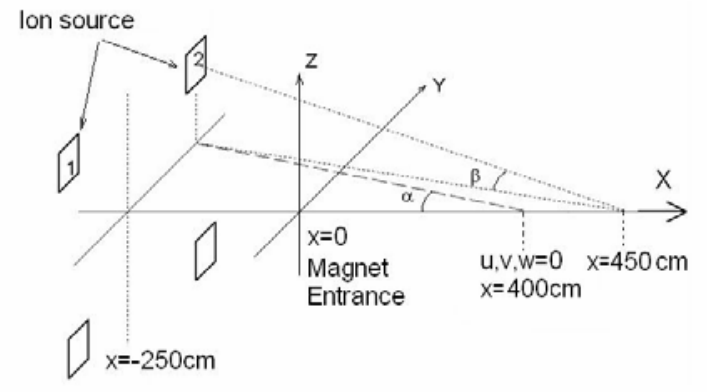

b) Positions of ion sources (rectangular approximation of racetrack shaped sources) compared to box axis. u, v, w see Fig. 1 


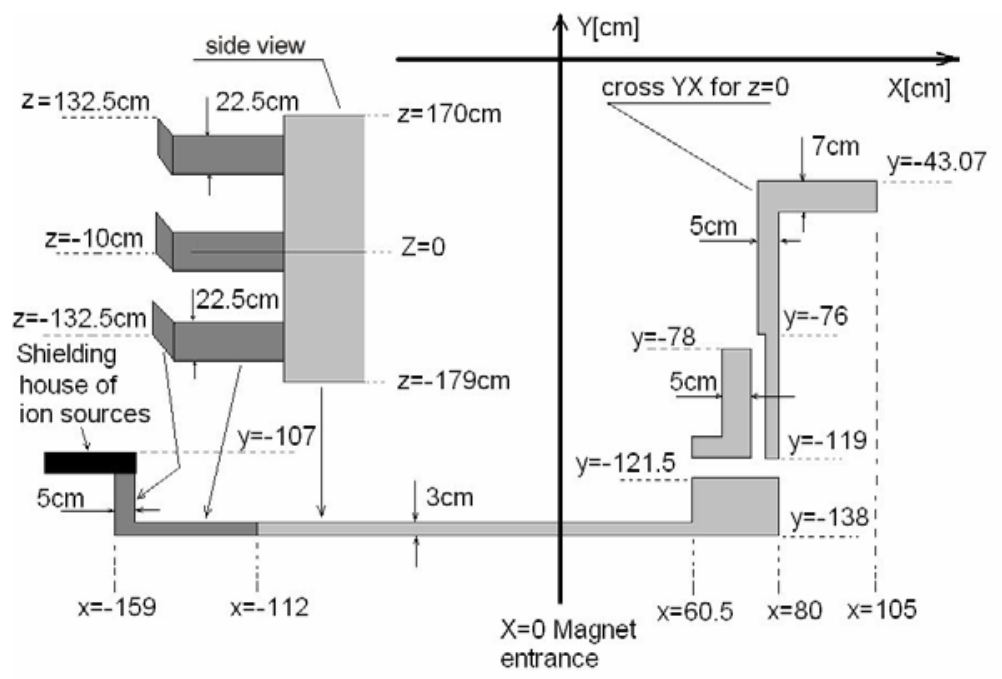
lations Mid plane view $(z=0)$ with inshielding house. Only a part of the ion source shielding house (where it is connected to the box shielding) is shown. PROFI coordinates are not to scale (but dependent on the size of the cells of calculations).
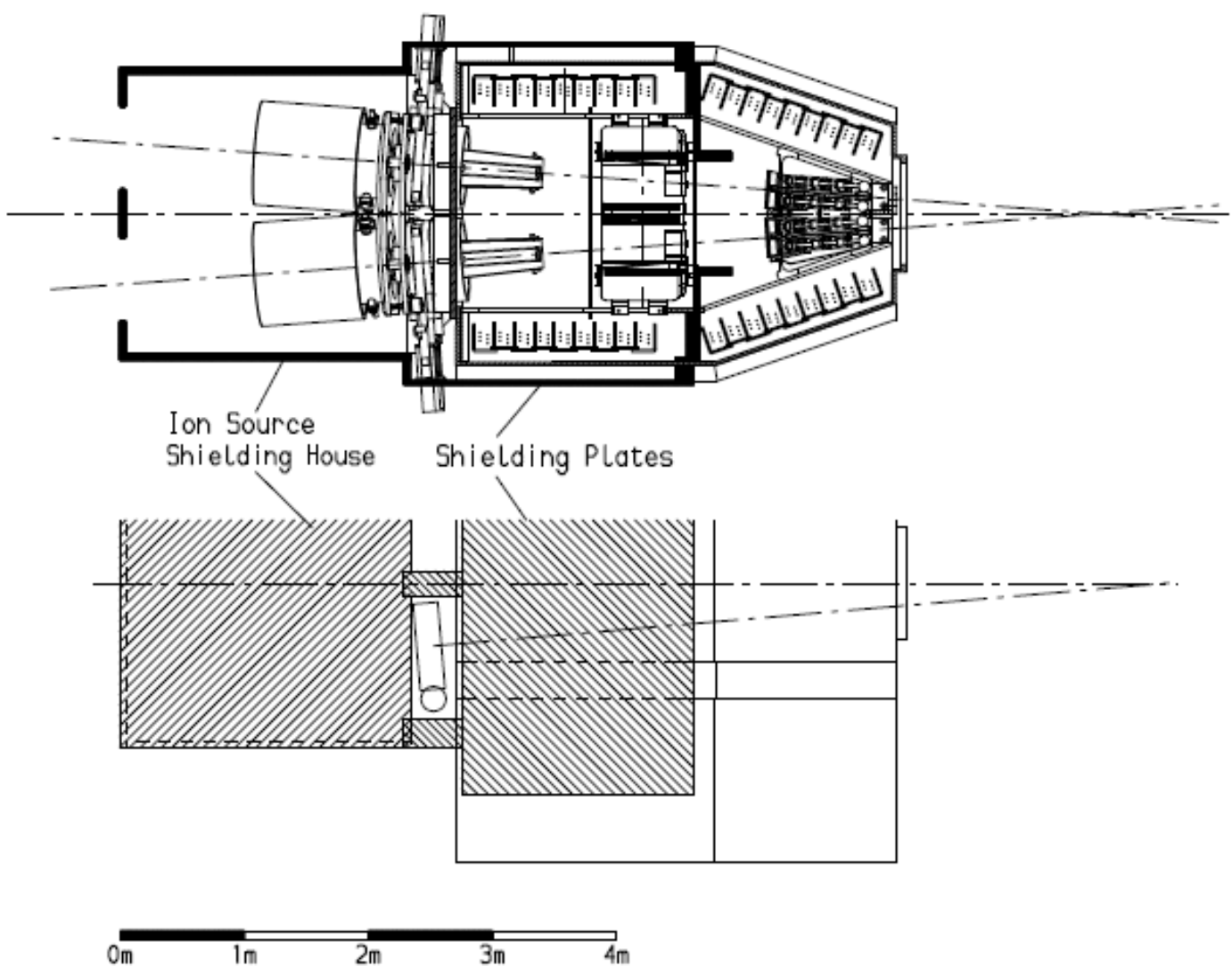

Fig. 4b) W7-X NBI box with technically possible magnetic shielding, upper sketch: mid plane cut, lower sketch: side view of lower half 


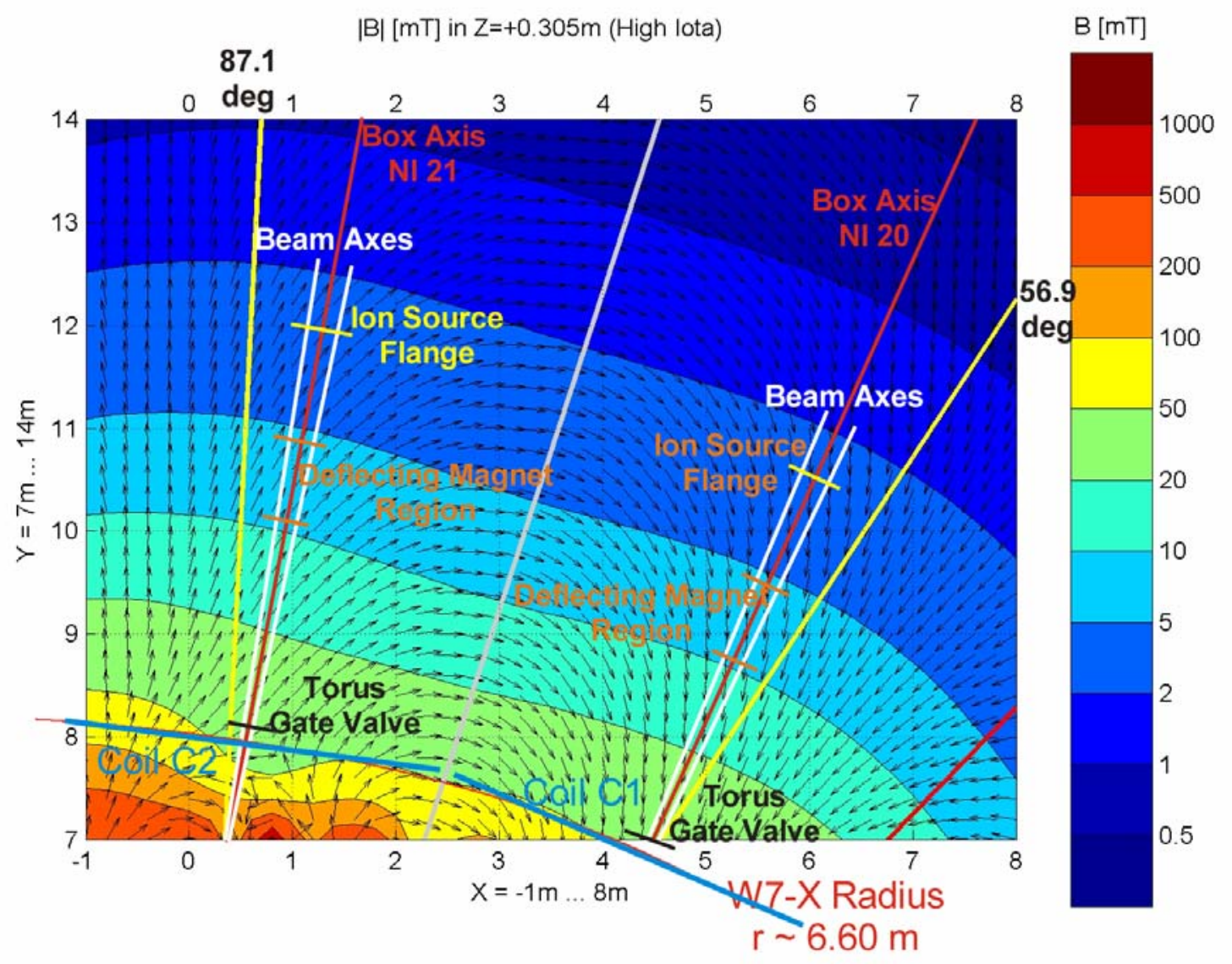

Fig. 5 a) W7-X hi iota field with the approximate locations of the planar coils, box axes, ion source flanges, etc.
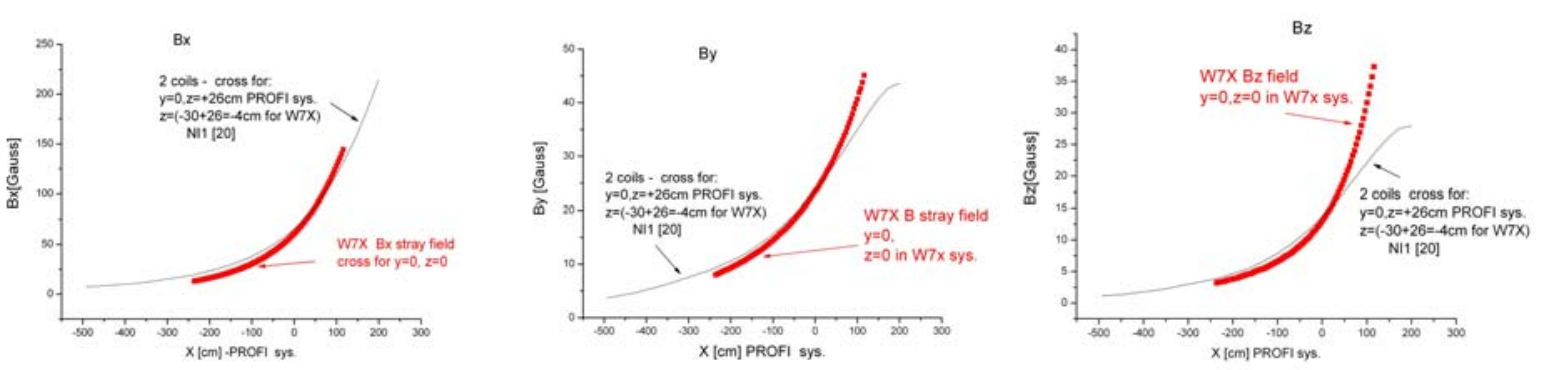

Fig. 5b) Comparison of calculated W7-X field (hi iota) to approximation by 2 planar coils: Red: W7X Bx, By, Bz fields calculated from the W7-X file: bfd+z000- hi. data Black: Bx, By, Bz values calculated from 2 coils 


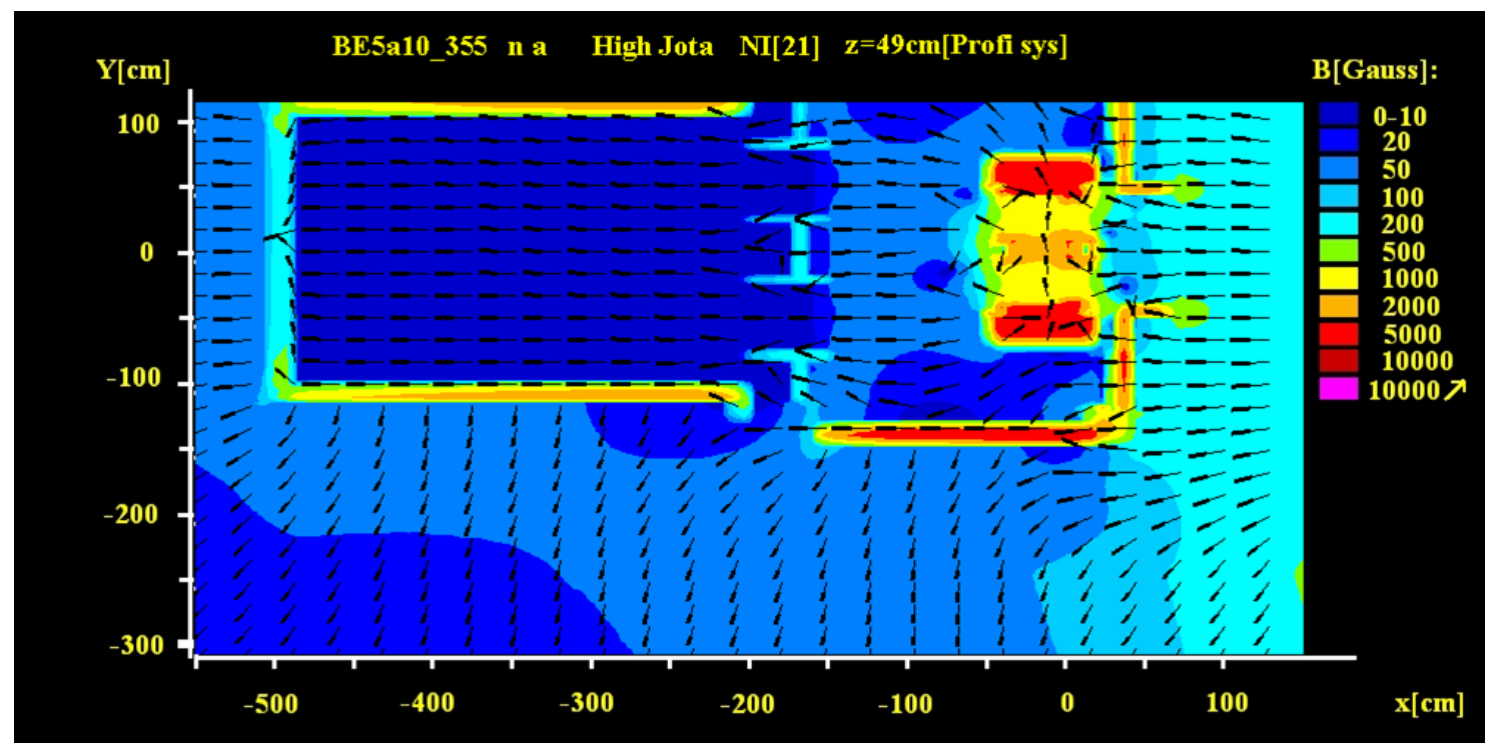

Fig. 6 a) NI 21 hi iota, magnetic field, colour code see fig. 2

\section{BE4a11_NI21_355na High Jota Main ID}

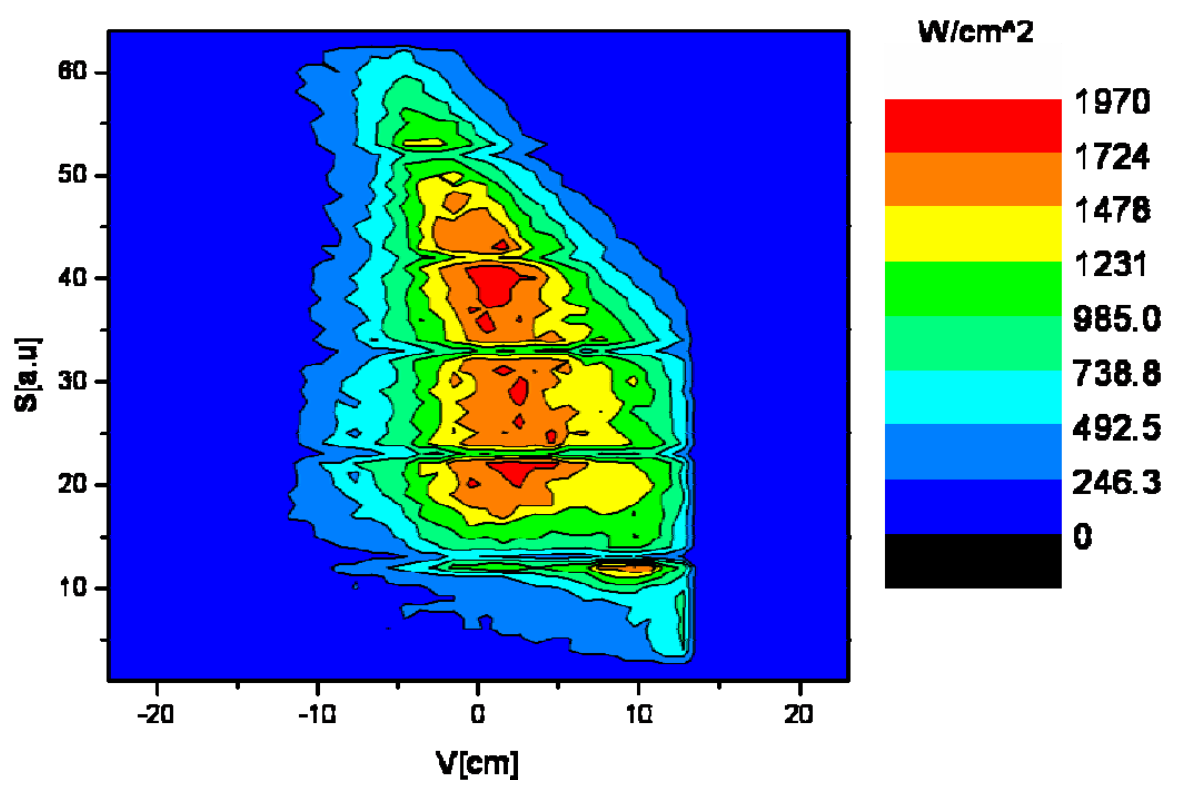

Fig. 6 b) NI 21 hi iota, power load on the six actively cooled panels of the main ion dump 


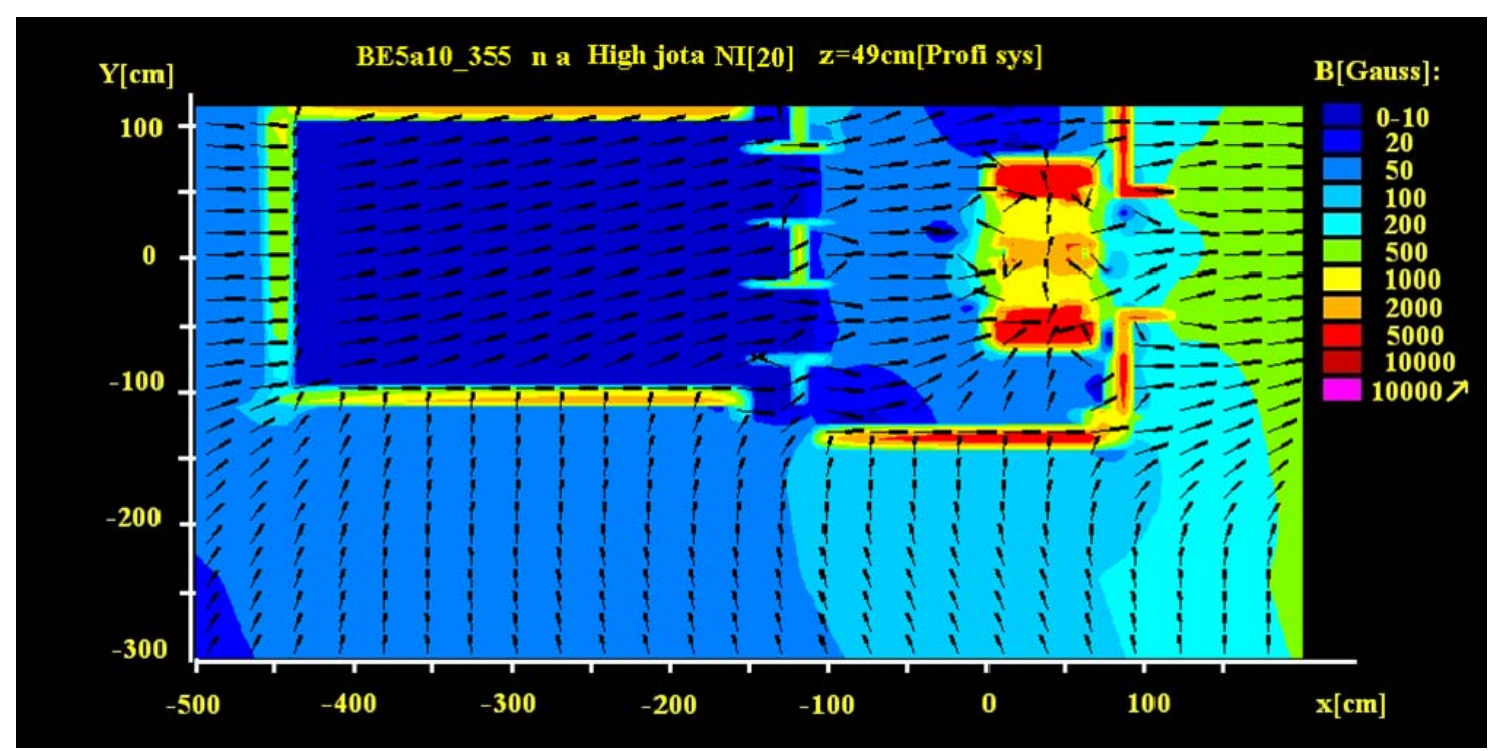

Fig. 7 a) NI 20, hi iota, magnetic field, colour code, see fig. 2

BE2b_N120_355na NI[20] High Jota Main ID

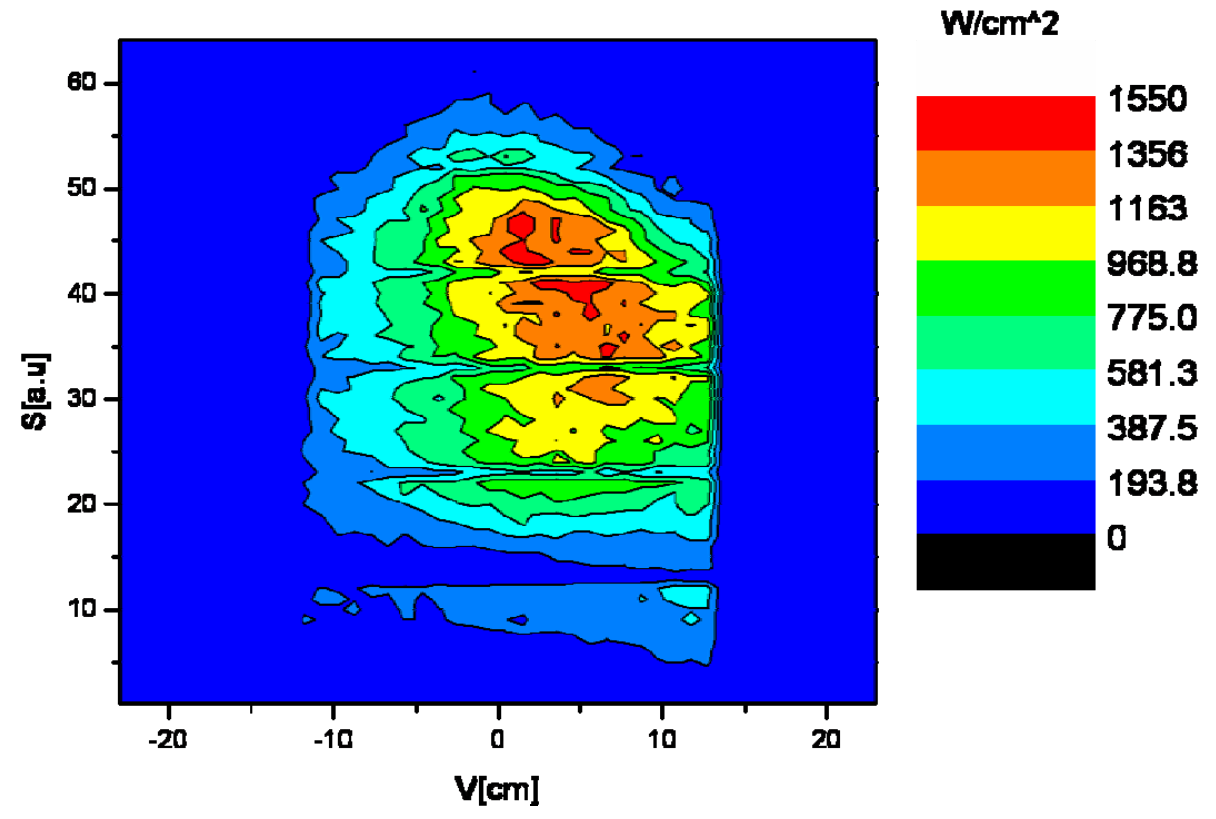

Fig. 7 b) NI 20, hi iota, power load on the six actively cooled panels of the main ion dump 


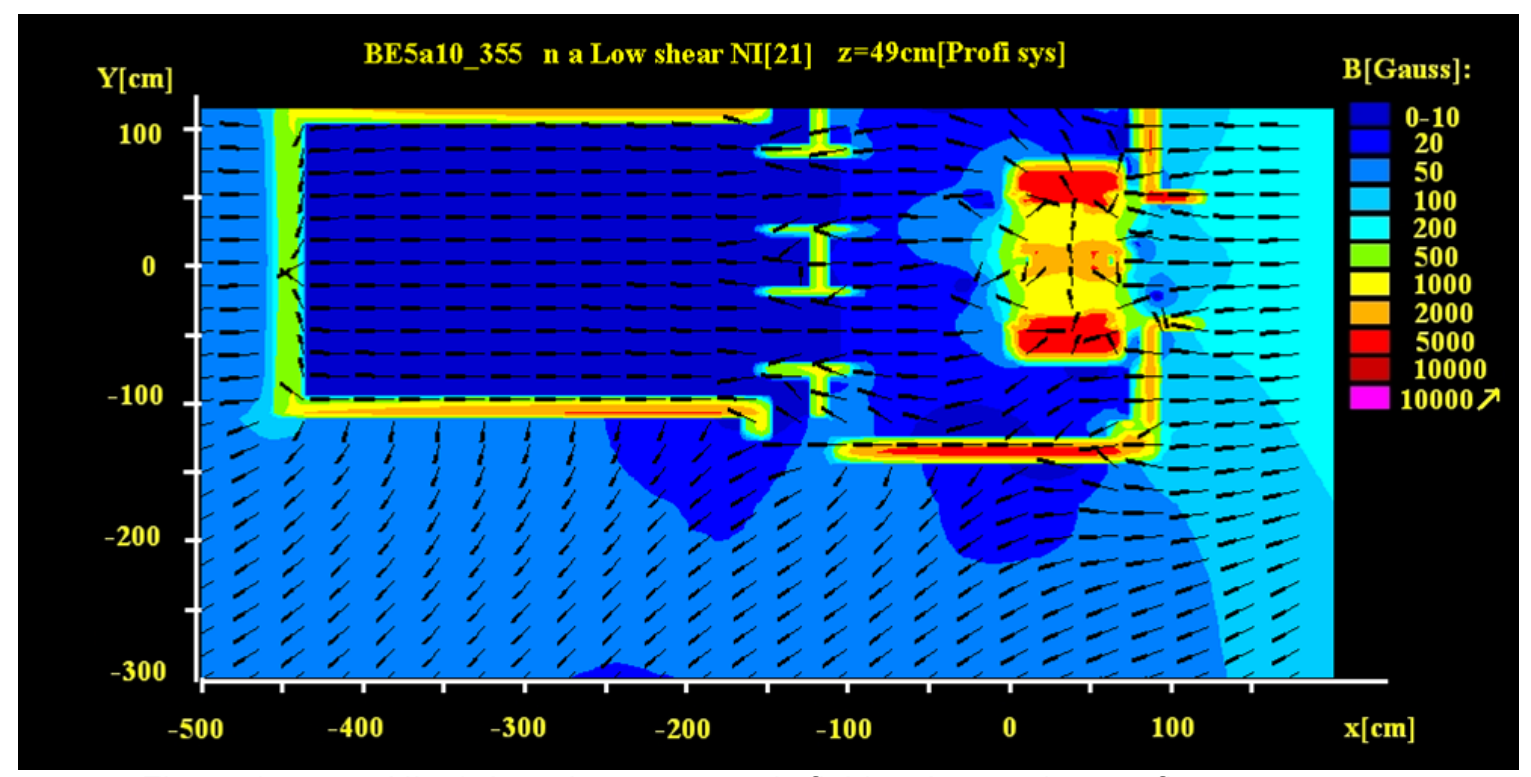

Fig. 8 a) $\quad$ NI 21, low shear, magnetic field, colour code, see fig. 2

BE5a10_355na -NI[21] - Low shear Main ID

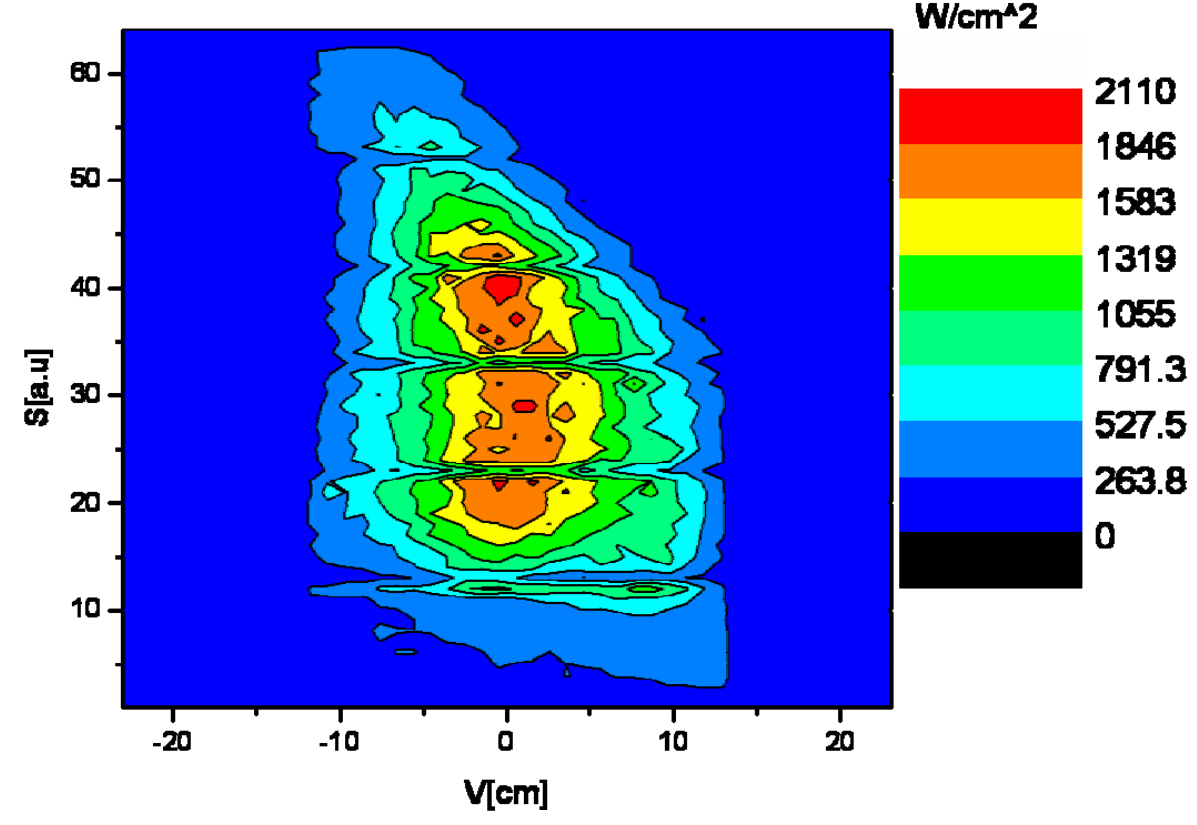

Fig. 8 b) NI 21, low shear, power load on the six actively cooled panels of the main ion dump 